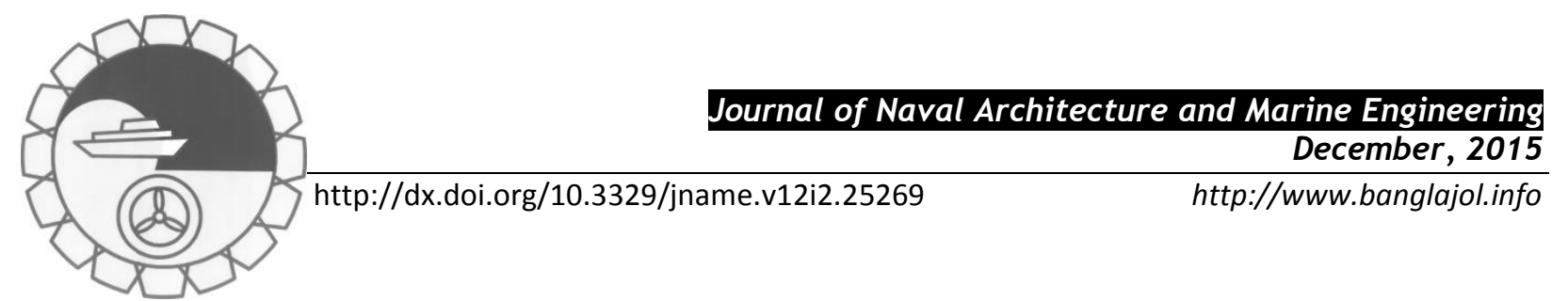

\title{
CASSON FLUID FLOW OVER A VERTICAL CONE WITH NON- UNIFORM HEAT SOURCE/SINK AND HIGH ORDER CHEMICAL REACTION
}

\author{
D. Mythili ${ }^{1}$, R. Sivaraj ${ }^{1 *}$, M. M. Rashidi ${ }^{2,3}$ and Z. Yang ${ }^{2,3}$ \\ ${ }^{1}$ Fluid Dynamics Division, School of Advanced Sciences, VIT University, Vellore, Tamilnadu, 632014, India. \\ *Email: sivaraj.kpm@gmail.com \\ ${ }^{2}$ Shanghai Key Lab of Vehicle Aerodynamics and Vehicle Thermal Management Systems, Tongji University, \\ 4800 Cao An Rd., Jiading, Shanghai 201804, China. \\ ${ }^{3}$ ENN-Tongji Clean Energy Institute of Advanced Studies, Shanghai, China.
}

\begin{abstract}
:
The present investigation deals with the study of unsteady, free convective Casson fluid flow over a vertical cone saturated with porous medium in the presence of non-uniform heat source/sink, high order chemical reaction and cross diffusion effects. The numerical computation for the governing equations has been performed using an implicit finite difference method of Crank-Nicolson type. The influence of various physical parameters on velocity, temperature and concentration distributions is illustrated graphically and the physical aspects are discussed in detail. Results indicate that temperature dependent heat source/sink plays a vital role on controlling the heat transfer however the surface-dependent heat source/sink also has notable influence on the heat transfer characteristics. It is to be noted that high order chemical reaction has the tendency to dilute the influence of chemical reaction parameter on the species concentration.
\end{abstract}

Keywords: Casson fluid, porous medium, non-uniform heat source/sink, high order chemical reaction, cross diffusion effects.

\section{NOMENCLATURE}

$r$ - dimensional local radius of the cone

$K_{1}$ - dimensional porous permeability parameter

$K$ - permeability coefficient of porous medium

A - co-efficient of space dependent heat

source/sink

$B$ - co-efficient of temperature dependent heat source/sink

$C_{S}$ - concentration susceptibility

$K_{T}$ - thermal diffusion ratio

$T_{m}$ - mean fluid temperature

$G r_{T}$ - thermal Grashof number

$N$ - buoyancy ratio parameter

\author{
$P_{r} \quad$ - Prandtl number \\ $D_{u}$ - Dufour number \\ $S_{c}$ - Schmidt number \\ $K_{r}$ - chemical reaction parameter \\ $S_{r} \quad$ - Soret number \\ $K_{R}$ - dimensional chemical reaction parameter \\ $l$ - order of chemical reaction (natural number) \\ Greek symbols \\ $\beta$ - Casson fluid parameter \\ $\beta_{T}$ - thermal expansion coefficient \\ $\beta_{C}$ - concentration expansion coefficient
}

\section{Introduction}

Casson fluid is one of the most significant non-Newtonian fluid which reflects the shear thinning property. It is interesting to note that Casson fluid asymptotically exhibits the behavior of Newtonian fluid when the shear stress increases to a level much higher than the yield stress. It was proposed by Casson in 1959 to predict the flow behavior of pigment-oil suspensions of printing ink. Now a days, Casson fluid model is widely accepted to depict the rheology of blood flow owing to the fact that yield stress of blood is a function of the volume fraction of red blood cells. Food substances such as jelly, tomato sauce, honey, soup, concentrated fruit juices are classic examples of Casson fluid. Many researchers such as Abolbashari et al. (2015, Animasaun (2015), Mukhopadhyay and Vajravelu (2013), Prasad et al. (2013) have shown interest in studying the characteristics of Casson fluid in various physical situations.

Coupled heat and mass transfer by natural convection in a fluid saturated with porous medium has many important applications in environmental, geophysical and industrial problems. These include the migration of moisture in fibrous insulation, casting and welding in manufacturing processes, solar power and diffusion of 
medicine in blood veins (Das, 2009, Raju and Varma, 2014, Sivaraj and RushiKumar, 2013). The analysis of heat generation/absorption effect in temperature field plays a vital role when the moving fluids are undergoing exothermic and/or endothermic chemical reaction which finds application in nuclear energy, convection in Earth's mantle, fire and combustion modeling. In the literature, many of the researchers have shown interest to examine the heat transfer characteristics with the influence of temperature dependent heat source/sink when a notable temperature difference is observed between the surface of solid body and ambient fluid. However, it is widely accepted that the consideration of surface dependent heat source/sink in addition to the temperature dependent heat source/sink aids to increase the accuracy of the heat transfer. Some mathematical models are proposed in which the volumetric rate of heat generation/absorption has been assumed to be any one of the following: constant, function of space variables, frictional heating and the expansion effect. Nandeppanavar et al. (2011), Okedoye (2014), Pal and Mondal (2010), Rahman et al. (2009) have studied and reported the significance of non-uniform heat source/sink in controlling the heat transfer in the boundary layer region.

The study of heat generation/absorption effects in fluids is important in view of several physical problems such as fluids undergoing chemical reactions. Thus in many industrial processes involving flow, heat and mass transfer over a surface, the diffusing species can be generated or absorbed due to some kind of chemical reaction with the ambient fluid which can greatly affect the flow and mass transfer characteristics. Processes involving the mass transfer effect have been recognized as important mainly in chemical processing equipments. The species generation in a homogeneous reaction is analogous to internal source of heat generation. In contrast, a heterogeneous reaction takes place in a restricted region or within the boundary of a phase. A chemical reaction is said to be of the first order and homogeneous if its rate is directly proportional to the concentration and it is a single-phase volume reaction. In addition, diffusing species generation or consumption caused by the reaction can be proportional to the $l^{\text {th }}$ order concentration difference between the surface and ambient fluid where $l$ is a natural number. Convective heat and mass transfer with chemical reaction plays an important role in meteorological phenomena, burning of haystacks, spray drying of milk, fluidized bed catalysis and cooling towers (Alam et al., 2009, Rahman and Al-Lawatia, 2010, Rashidi et al., 2012, RushiKumar and Sivaraj, 2013, Sivaraj and RushiKumar, 2013).

Soret effect has notable influence when large density differences exist in the flow regime. For example, Soret effect can be significant when species are introduced at a surface in fluid domain, with a density lower than the surrounding fluid. The Soret effect is also utilized for isotope separation and in mixture between gases with very light molecular weight $(\mathrm{H} 2, \mathrm{He})$ and of medium molecular weight (N2, air). In many liquid mixtures, the Dufour effect is inoperative, but this may not be the case in gasses. The Soret and Dufour effects may become significant in many practical applications pertaining to the areas of geosciences and chemical engineering where the temperature and concentration gradients are high (Postelnicu, 2004, RamReddy, 2013, RushiKumar and Sivaraj, 2013).

In view of the above the investigations and numerous possible applications, we intend to analyze the influence of cross-diffusion effects on unsteady, free convective Casson fluid flow over a vertical cone saturated with porous medium. In literature, many studies have reported the heat and mass transfer characteristics with the influence of heat source/sink and chemical reaction but the influence of surface dependent heat source/sink and order of chemical reaction have been ignored. It is apparent that the accuracy of heat and mass transfer is increased when the surface dependent heat source/sink and order of chemical reaction are considered in addition to the temperature dependent heat source/sink and chemical reaction. The governing equations are solved using the Crank - Nicolson method and the features of the flow, heat and mass transfer characteristics are presented through graphs and the physical aspects are discussed in detail.

\section{Mathematical Formulation}

Consider a two-dimensional, unsteady, free convective flow of an incompressible Casson fluid over a vertical cone (with half angle $\alpha$ ) saturated with porous medium of a coordinate system as shown in Fig. 1.

It is assumed that the medium is isotropic, neither radiative nor dissipative. The $x$-axis is measured along surface of the cone and $y$-axis is measured perpendicular it. $T_{w}$ and $C_{w}$ are respectively, the temperature and concentration at the wall $y=0$ at any time $t$ are assumed to be higher than the respective ambient values $T_{\infty}$ and $C_{\infty}$ of the cone. The porous is assumed to be uniform (that is, it has a constant porosity and permeability). The flow is influenced by non-uniform heat source/sink, high order chemical reaction and Soret and Dufour effects. The basic two-dimensional equations governing the conservation of continuity, momentum, energy and 
mass diffusion for Casson fluid using the above assumptions along with Boussinesq approximations, can be written as:

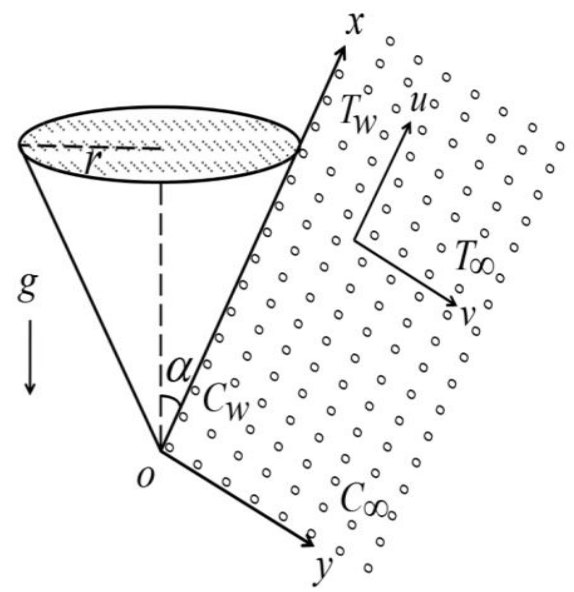

Fig. 1: Flow geometry of the problem

$\frac{\partial(r u)}{\partial x}+\frac{\partial(r v)}{\partial y}=0$

$\frac{\partial u}{\partial t^{*}}+u \frac{\partial u}{\partial x}+v \frac{\partial u}{\partial y}=v\left[1+\frac{1}{\beta}\right] \frac{\partial^{2} u}{\partial y^{2}}-\frac{v}{K_{1}} u+g \beta_{T} \cos (\alpha)\left(T-T_{\infty}\right)+g \beta_{C} \cos (\alpha)\left(C-C_{\infty}\right)$

$\frac{\partial T}{\partial t^{*}}+u \frac{\partial T}{\partial x}+v \frac{\partial T}{\partial y}=\frac{k}{\rho C_{P}} \frac{\partial^{2} T}{\partial y^{2}}+\frac{q^{\prime \prime \prime}}{\rho C_{P}}+\frac{D K_{T}}{C_{S} C_{P}} \frac{\partial^{2} C}{\partial y^{2}}$

$\frac{\partial C}{\partial t^{*}}+u \frac{\partial C}{\partial x}+v \frac{\partial C}{\partial y}=D \frac{\partial^{2} C}{\partial y^{2}}-K_{R}\left(C-C_{\infty}\right)^{l}+\frac{D K_{T}}{T_{m}} \frac{\partial^{2} T}{\partial y^{2}}$

The appropriate initial and boundary conditions of the problem are

$t^{*} \leq 0: u=0, \quad v=0, \quad T=T_{\infty}, \quad C=C_{\infty}$ for all $x, y$

$t^{*}>0: u=0, \quad v=0, \quad T=T_{w}, \quad C=C_{w} \quad$ at $\quad y=0$

$u=0, \quad T=T_{\infty}, \quad C=C_{\infty} \quad$ at $\quad x=0$

$u \rightarrow 0, \quad T \rightarrow T_{\infty}, C \rightarrow C_{\infty}$ as $y \rightarrow \infty$

The non-uniform heat sink/source $q^{\prime \prime \prime}$ is modeled as (Pal and Mondal, 2010)

$q^{\prime \prime \prime}=\frac{k L^{2}}{\left(G r_{T}\right)^{1 / 2}}\left(\frac{A L}{v\left(G r_{T}\right)^{1 / 2}}\left(T_{w}-T_{\infty}\right) u+B\left(T-T_{\infty}\right)\right)$

Introducing the following non-dimensional quantities

$$
\begin{aligned}
& X=\frac{x}{L}, \quad Y=\frac{y}{L}\left(G r_{T}\right)^{1 / 4}, \quad R=\frac{r}{L}, \quad r=x \sin (\alpha), \quad U=\frac{u L}{v}\left(G r_{T}\right)^{-1 / 2}, \quad V=\frac{v L}{v}\left(G r_{T}\right)^{-1 / 4}, \\
& t=\frac{v t^{*}}{L^{2}}\left(G r_{T}\right)^{1 / 2}, \quad \theta=\frac{T-T_{\infty}}{T_{w}-T_{\infty}}, \quad \phi=\frac{C-C_{\infty}}{C_{w}-C_{\infty}}, \quad G r_{T}=\frac{g \beta_{T}\left(T_{w}-T_{\infty}\right) L^{3}}{v^{2}}, \quad P_{r}=\frac{\mu C_{P}}{k}, \\
& \frac{1}{K}=\frac{L^{2}}{K_{1}\left(G r_{T}\right)^{1 / 2}}, \quad N=\frac{\beta_{C}\left(C_{w}-C_{\infty}\right)}{\beta_{T}\left(T_{w}-T_{\infty}\right)}, \quad D_{u}=\frac{K_{T} D\left(C_{w}-C_{\infty}\right)}{v C_{S} C_{P}\left(T_{w}-T_{\infty}\right)}, \quad S_{c}=\frac{v}{D},
\end{aligned}
$$




$$
K_{r}=\frac{K_{R} L^{2}}{v\left(G r_{T}\right)^{1 / 2}}, \quad \mathrm{~S}_{r}=\frac{K_{T} D\left(T_{w}-T_{\infty}\right)}{v T_{m}\left(C_{w}-C_{\infty}\right)}
$$

In view of Eq. (7), the basic field of Eqs. (1) - (4) are expressed in non-dimensional form as

$$
\begin{aligned}
& \frac{\partial(R u)}{\partial X}+\frac{\partial(R v)}{\partial Y}=0 \\
& \frac{\partial U}{\partial t}+U \frac{\partial U}{\partial X}+V \frac{\partial U}{\partial Y}=\left[1+\frac{1}{\beta}\right] \frac{\partial^{2} U}{\partial Y^{2}}-\frac{1}{K} U+\cos (\alpha) \theta+N \cos (\alpha) \phi \\
& \frac{\partial \theta}{\partial t}+U \frac{\partial \theta}{\partial X}+V \frac{\partial \theta}{\partial Y}=\frac{1}{P_{r}} \frac{\partial^{2} \theta}{\partial Y^{2}}+\frac{1}{P_{r}}(A U+B \theta)+D_{u} \frac{\partial^{2} \phi}{\partial Y^{2}} \\
& \frac{\partial \phi}{\partial t}+U \frac{\partial \phi}{\partial X}+V \frac{\partial \phi}{\partial Y}=\frac{1}{S_{c}} \frac{\partial^{2} \phi}{\partial Y^{2}}-K_{r} \phi^{l}+S_{r} \frac{\partial^{2} \theta}{\partial Y^{2}}
\end{aligned}
$$

The appropriate initial and boundary conditions become

$$
\begin{aligned}
t \leq 0: & U=0, \quad V=0, \quad \theta=0, \quad \phi=0 \quad \text { for all } X, Y \\
t>0: & U=0, \quad V=0, \quad \theta=1, \quad \phi=1 \text { at } Y=0 \\
U & =0, \quad \theta=0, \quad \phi=0 \quad \text { at } X=0 \\
U & \rightarrow 0, \quad \theta \rightarrow 0, \quad \phi \rightarrow 0 \text { as } Y \rightarrow \infty
\end{aligned}
$$

The local skin Friction, Nusselt numbers and Sherwood number at the wall are

$$
\tau=-\left[1+\frac{1}{\beta}\right]\left(\frac{\partial U}{\partial Y}\right)_{Y=0}, \quad N u=-X\left(\frac{\partial \theta}{\partial Y}\right)_{Y=0}, \quad S h=-X\left(\frac{\partial \phi}{\partial Y}\right)_{Y=0}
$$

Average skin friction, Nusselt number and Sherwood number can be written as:

$$
\bar{\tau}=-\left[1+\frac{1}{\beta}\right]_{0}^{1} \int_{0}\left(\frac{\partial U}{\partial Y}\right)_{Y=0} d X, \quad \overline{N u}=-\int_{0}^{1}\left(\frac{\partial \theta}{\partial Y}\right)_{Y=0} d X, \quad \overline{S h}=-\int_{0}^{1}\left(\frac{\partial \phi}{\partial Y}\right)_{Y=0} d X
$$

\section{Finite Difference Numerical Solution}

The set of coupled non- linear differential Eqns. (8) - (11) subjected to the initial and boundary conditions (12) are solved by implicit finite difference scheme of Crank - Nicolson type. The discretized form of the continuity, velocity, temperature and concentration equations are given as follows.

$$
\begin{aligned}
& \frac{U_{i, j}^{n+1}-U_{i-1, j}^{n+1}+U_{i, j}^{n}-U_{i-1, j}^{n}+U_{i, j-1}^{n+1}-U_{i-1, j-1}^{n+1}+U_{i, j-1}^{n}-U_{i-1, j-1}^{n}}{4 \Delta X}+\frac{V_{i, j}^{n+1}-V_{i, j-1}^{n+1}+V_{i, j}^{n}-V_{i, j-1}^{n}}{2 \Delta Y} \\
& \quad+\frac{U_{i, j}^{n+1}+U_{i, j-1}^{n+1}+U_{i, j}^{n}+U_{i, j-1}^{n}}{4 i \Delta X}=0 \\
& \frac{U_{i, j}^{n+1}-U_{i, j}^{n}}{\Delta t}+\frac{U_{i, j}^{n}\left(U_{i, j}^{n+1}-U_{i-1, j}^{n+1}+U_{i, j}^{n}-U_{i-1, j}^{n}\right)}{2 \Delta X}+\frac{V_{i, j}^{n}\left(U_{i, j+1}^{n+1}-U_{i, j-1}^{n+1}+U_{i, j+1}^{n}-U_{i, j-1}^{n}\right)}{4 \Delta Y} \\
& =\left[1+\frac{1}{\beta}\right] \frac{U_{i, j-1}^{n+1}-2 U_{i, j}^{n+1}+U_{i, j+1}^{n+1}+U_{i, j-1}^{n}-2 U_{i, j}^{n}+U_{i, j+1}^{n}}{2(\Delta Y)^{2}}-\frac{1}{K} \frac{U_{i, j}^{n+1}+U_{i, j}^{n}}{2} \\
& +\cos (\alpha) \frac{\theta_{i, j}^{n+1}+\theta_{i, j}^{n}}{2}+N \cos (\alpha) \frac{\phi_{i, j}^{n+1}+\phi_{i, j}^{n}}{2}
\end{aligned}
$$




$$
\begin{aligned}
& \frac{\theta_{i, j}^{n+1}-\theta_{i, j}^{n}}{\Delta t}+\frac{U_{i, j}^{n}\left(\theta_{i, j}^{n+1}-\theta_{i-1, j}^{n+1}+\theta_{i, j}^{n}-\theta_{i-1, j}^{n}\right)}{2 \Delta X}+\frac{V_{i, j}^{n}\left(\theta_{i, j+1}^{n+1}-\theta_{i, j-1}^{n+1}+\theta_{i, j+1}^{n}-\theta_{i, j-1}^{n}\right)}{4 \Delta Y} \\
& =\frac{1}{P_{r}} \frac{\theta_{i, j-1}^{n+1}-2 \theta_{i, j}^{n+1}+\theta_{i, j+1}^{n+1}+\theta_{i, j-1}^{n}-2 \theta_{i, j}^{n}+\theta_{i, j+1}^{n}+\frac{1}{2(\Delta Y)^{2}}\left(A\left(U_{i, j}^{n+1}+U_{i, j}^{n}\right)+B\left(\theta_{i, j}^{n+1}+\theta_{i, j}^{n}\right)\right)}{2(\Delta Y)^{2}} \\
& +D_{u} \frac{\phi_{i, j-1}^{n+1}-2 \phi_{i, j}^{n+1}+\phi_{i, j+1}^{n+1}+\phi_{i, j-1}^{n}-2 \phi_{i, j}^{n}+\phi_{i, j+1}^{n}}{2 \Delta X} \\
& \frac{\phi_{i, j}^{n+1}-\phi_{i, j}^{n}}{\Delta t}+\frac{U_{i, j}^{n}\left(\phi_{i, j}^{n+1}-\phi_{i-1, j}^{n+1}+\phi_{i, j}^{n}-\phi_{i-1, j}^{n}\right)}{2 \Delta X}+\frac{V_{i, j}^{n}\left(\phi_{i, j+1}^{n+1}-\phi_{i, j-1}^{n+1}+\phi_{i, j+1}^{n}-\phi_{i, j-1}^{n}\right)}{4 \Delta Y} \\
& =\frac{1}{S_{c}} \frac{\phi_{i, j-1}^{n+1}-2 \phi_{i, j}^{n+1}+\phi_{i, j+1}^{n+1}+\phi_{i, j-1}^{n}-2 \phi_{i, j}^{n}+\phi_{i, j+1}^{n}}{2(\Delta Y)^{2}}-K_{r}\left(\frac{\phi_{i, j}^{n+1}+\phi_{i, j}^{n}}{2}\right)^{l} \\
& +S_{r} \frac{\theta_{i, j-1}^{n+1}-2 \theta_{i, j}^{n+1}+\theta_{i, j+1}^{n+1}+\theta_{i, j-1}^{n}-2 \theta_{i, j}^{n}+\theta_{i, j+1}^{n}}{2(\Delta Y)^{2}}
\end{aligned}
$$

The finite difference notation for initial and boundary conditions are

$$
\begin{aligned}
& n \leq 0: \quad U_{i, j}^{n}=0, \quad V_{i, j}^{n}=0, \quad \theta_{i, j}^{n}=0, \quad \phi_{i, j}^{n}=0 \\
& n>0: \quad U_{i, 0}^{n}=0, \quad V_{i, 0}^{n}=0, \quad \theta_{i, 0}^{n}=1, \quad \phi_{i, 0}^{n}=1 \\
& U_{0, j}^{n}=0, \quad \theta_{0, j}^{n}=0, \quad \phi_{0, j}^{n}=0 \\
& U_{i, j}^{n} \rightarrow 0, \quad \theta_{i, j}^{n} \rightarrow 0, \quad \phi_{i, j}^{n} \rightarrow 0 \quad \text { as } \quad j \rightarrow \infty
\end{aligned}
$$

Here the subscript $i$ designates the grid-point with $X$ coordinate $\sum_{i=0}^{I} \Delta X_{i}$; subscript $j$ designates the grid-point with $Y$ coordinate $\sum_{j=0}^{J} \Delta Y_{j}$ and the superscript $n$ designates a value of time. We consider a rectangular region with $X$ varying from 0 to 1 and $\mathrm{Y}$ varying from 0 to 22 , where $\mathrm{Y}_{\max }$ is regarded as $Y \rightarrow \infty$. It is ensured that $\mathrm{Y}_{\max }$ lies well outside the dynamic, thermal and mass diffusion boundary layers. The maximum of $\mathrm{Y}$ is estimated at 22 after some preliminary investigations, so that the initial and boundary conditions of Eqn. (12) are satisfied. The mesh spacing in the $X$ and $Y$ directions $\Delta X=0.05, \Delta Y=0.25$ and time interval $\Delta t=0.01$ are selected to obtain the tolerance limit within $10^{-5}$. Computations are repeated until the steady state is reached. A convergence criterion based on the relative difference between the two consecutive iteration values is employed. When the difference reaches less than $10^{-5}$ at all grid points, the solution is assumed to have converged and the iterative process is terminated. The scheme is unconditionally stable. The local truncation error is $O\left(\Delta t^{2}+\Delta X^{2}+\Delta Y^{2}\right)$ and it tends to zero as $\Delta t, \Delta X$ and $\Delta Y$ tend to zero. It follows that the Crank-Nicolson Method is compatible. Stability and compatibility ensure the convergence.

\section{Result and Discussion}

In order to get a clear insight of this mathematical model, the influence of various pertinent parameters on flow, heat and mass transfer characteristics have been analyzed and the results are furnished in form of Figs. 2 - 20 and Tables $1-2$. The following choice of values for governing parameters are adopted based on the previous investigations, $\beta=1, K=1, N=0.5, P_{r}=2.97, A=1, B=1, D_{u}=0.15, S_{c}=0.22, K_{r}=1$ and $S_{r}=0.4$; unless otherwise stated. 


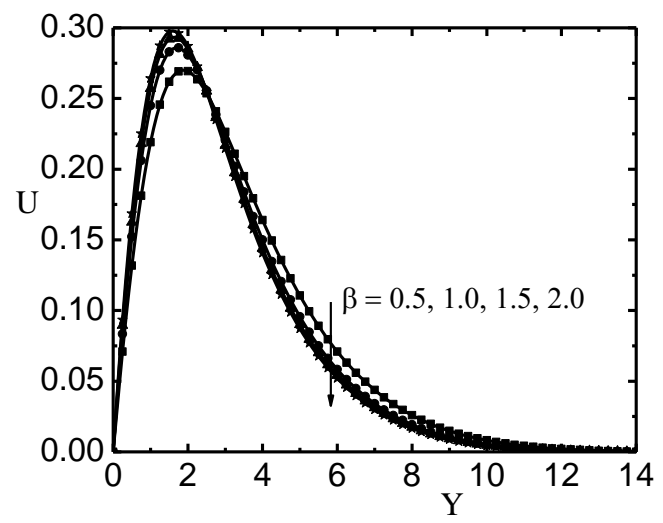

Fig. 2: Effect of Casson fluid parameter on velocity distribution

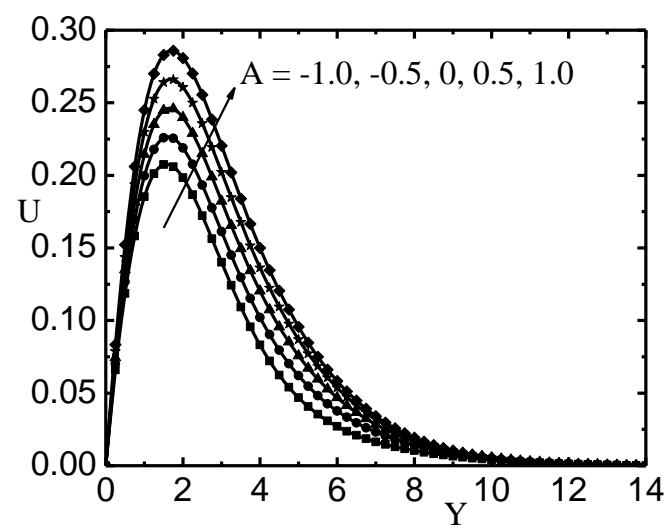

Fig. 4: Effect of space-dependent heat source/sink Fig. 5: Effect of temperature-dependent heat parameter on velocity distribution

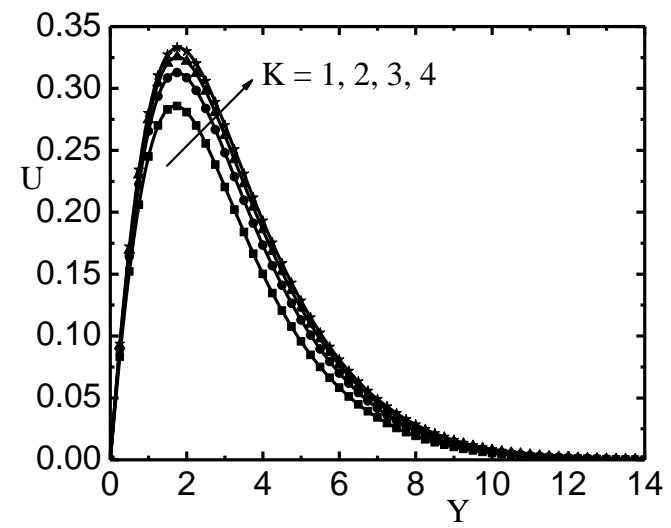

Fig. 3: Effect of porous permeability parameter on velocity distribution

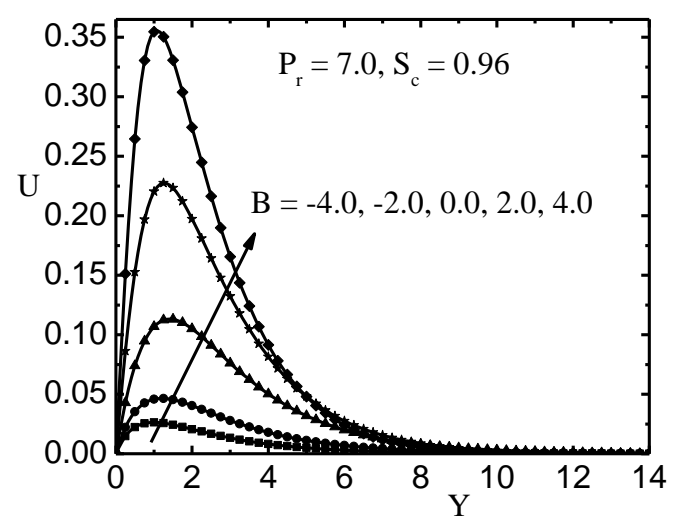
source/sink parameter on velocity distribution

Figs. 2-6 render the velocity profiles for different values of $\beta, K, A, B$, and $D_{u} \& S_{r}$ respectively. Fig. 2 elucidates that velocity profiles decrease with an increase in the values of $\beta$ little away from the cone $(\mathrm{Y} \approx 4)$. The rise in $\beta$ is used to dilute the strength of yield stress $P_{y}$ of the Casson fluid which enhances the value of plastic dynamic viscosity $\mu_{\mathrm{B}}$ and causing the resistance in fluid flow. An increase in porous permeability parameter accelerates the velocity along the boundary layer by deteriorating the resistivity of porous medium which is demonstrated in Fig. 3. The influence of space and temperature dependent heat source $(A>0 ; B>0)$ and sink $(A<0 ; B<0)$ on the velocity profiles is presented in Figs. 4 and 5, respectively. It is clear from these figures that increasing the values of heat sources enhance the velocity profiles by heating the fluid whereas decreasing the values of heat sinks reduce the flow velocity by cooling the fluid. It is observed from Fig. 6 that an increase in Dufour number with a decrease in Soret number is used to increase the velocity profiles.

Figs. 7 - 10 are plotted to show the influence of $P_{r}, A, B$ and $D_{u} \& S_{r}$ on temperature profiles, respectively. It is apparent from Fig. 7 that increasing the values of Prandtl number is used to decrease the heat transfer of the fluid. This is due to the fact that a higher Prandtl number fluid has relatively low thermal conductivity. The reduction in conductivity of fluid dilutes the thermal boundary layer thickness. The values of the Prandtl number are selected to represent the presence of air $\left(P_{r}=0.71\right)$, methyl chloride $\left(P_{r}=2.97\right)$, sulfur dioxide $\left(P_{r}=4.24\right)$ and water at $20^{\circ}\left(P_{r}=7\right)$. 


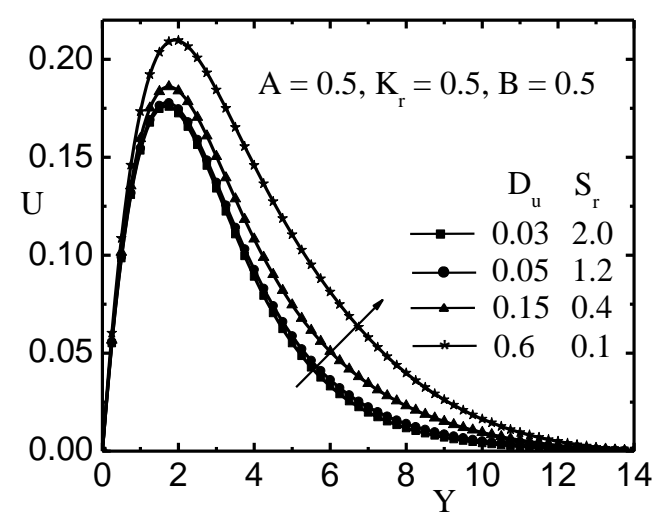

Fig. 6: Effect of Dufour and Soret number on velocity distribution

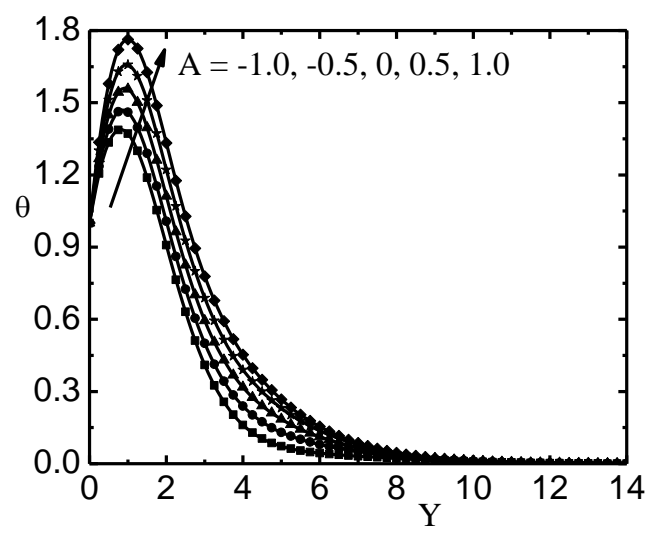

Fig. 8: Effect of space-dependent heat source/sink parameter on temperature distribution

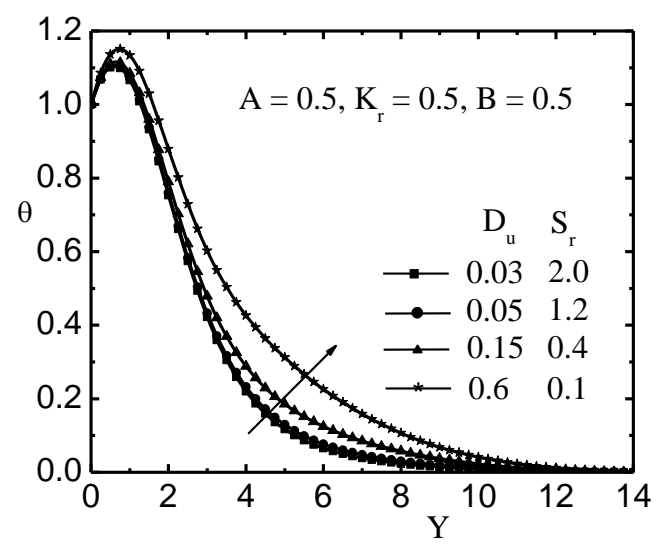

Fig. 10: Effect of Dufour and Soret on temperature distribution

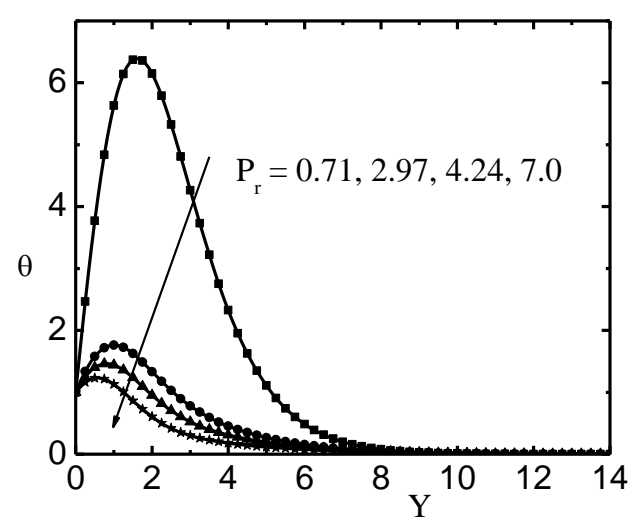

Fig. 7: Effect of Prandtl number on temperature distribution

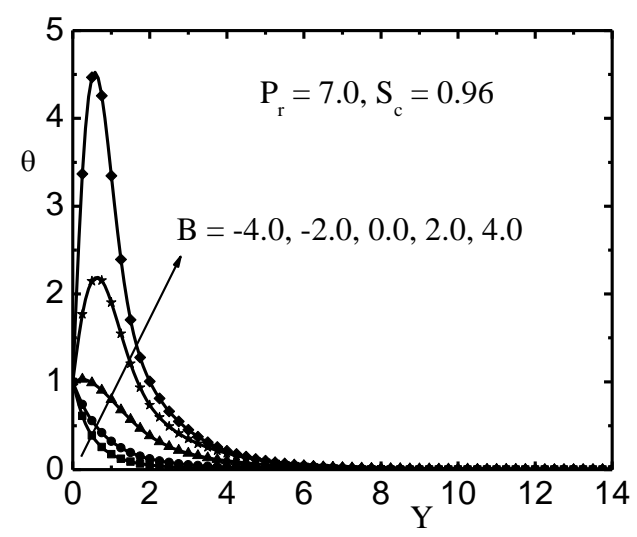

Fig. 9: Effect of temperature-dependent heat source/sink parameter on temperature distribution

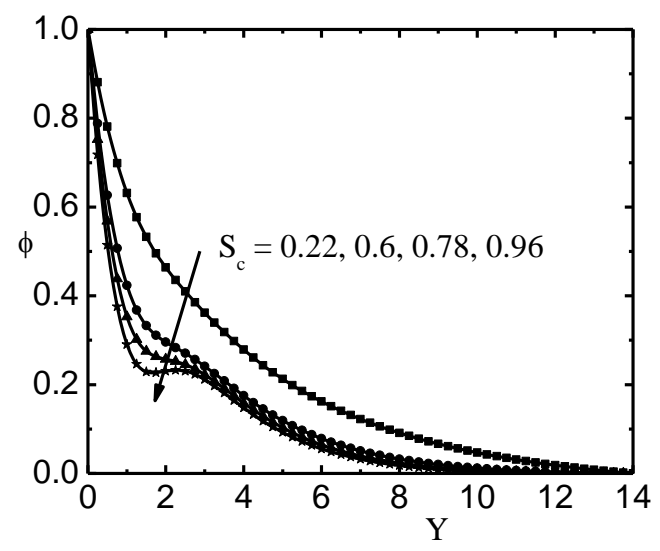

Fig. 11: Effect of Schmidt number on concentration distribution 


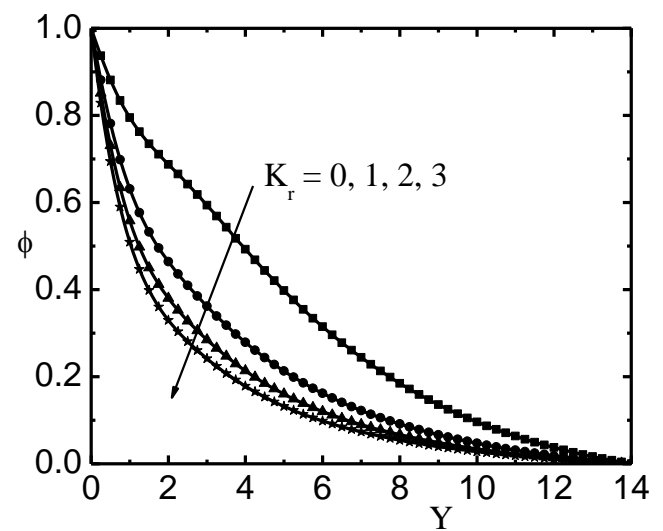

Fig. 12: Effect of chemical reaction parameter on Fig. 13: Effect of order of chemical reaction parameter concentration distribution

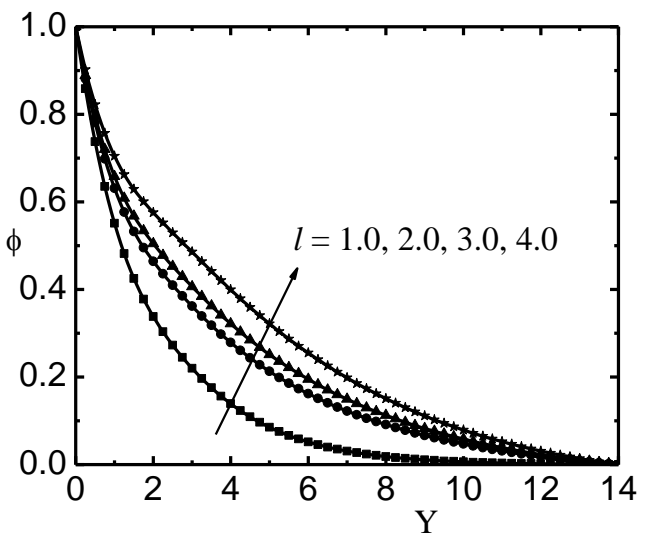

on concentration distribution

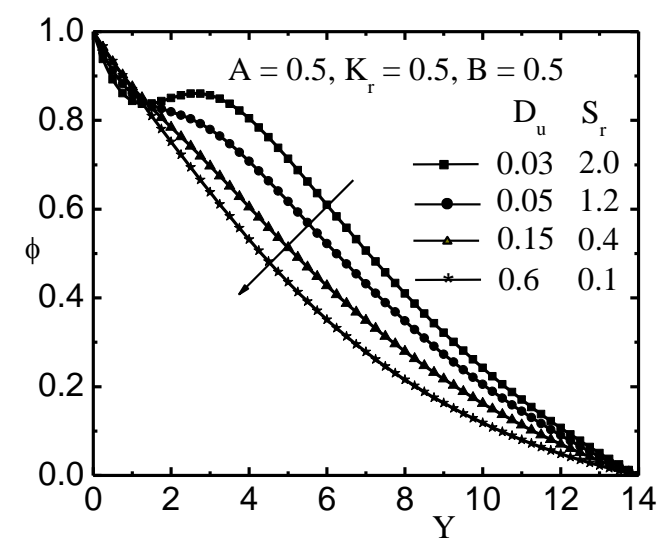

Fig. 14: Effect of Dufour and Soret number onconcentration distribution

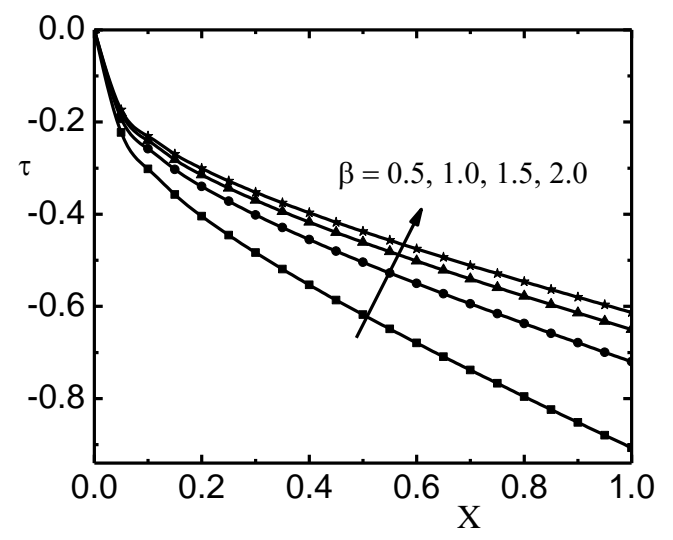

Fig. 15: Effect of Casson fluid parameter on the local skin friction

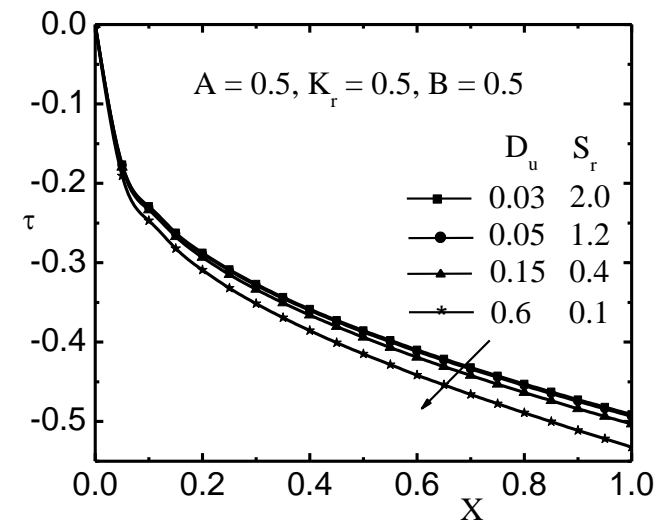

Fig. 16: Effect of Dufour and Soret on the skin friction 


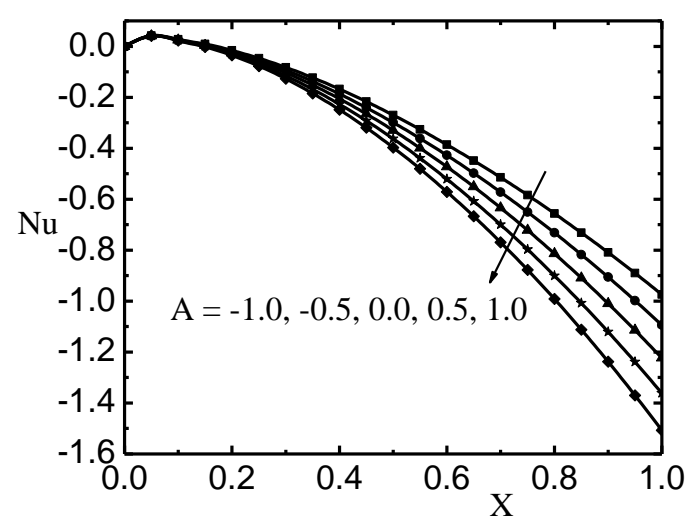

Fig. 17: Effect of space-dependent heat source heat parameter on the local Nusselt number

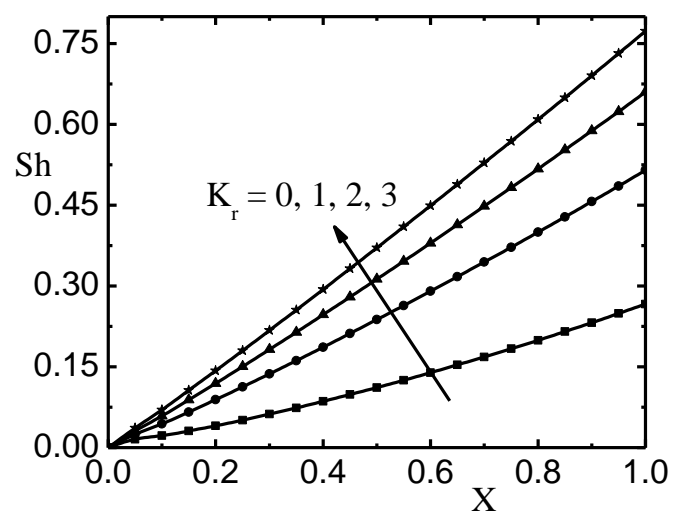

Fig. 19: Effect of chemical reaction parameter on the local Sherwood number

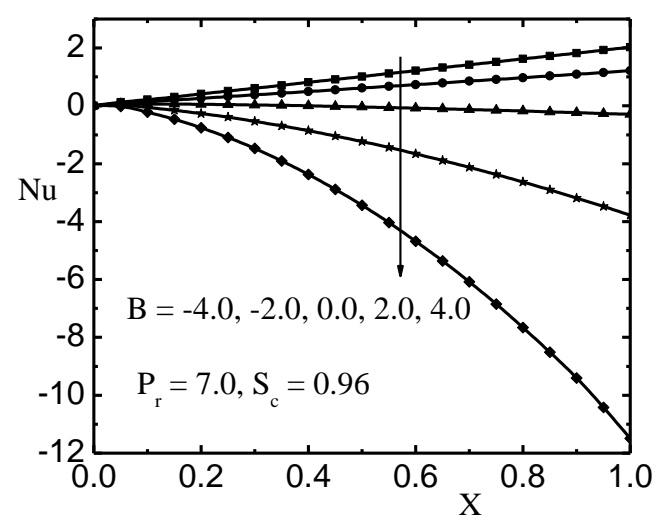

Fig. 18: Effect of temperature-dependent source or sink or sink parameter on the local Nusselt number

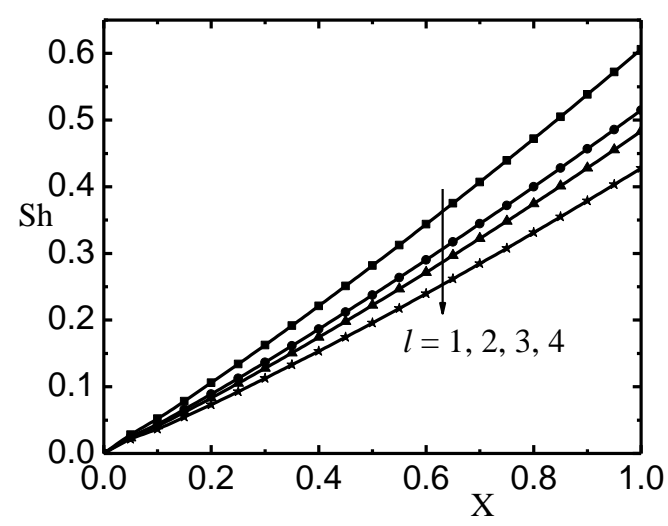

Fig. 20: Effect of order of chemical reaction parameter on the local Sherwood number

It is observed from Figs. 8 and 9 that the thermal boundary layer generates the energy for increasing the values of $A>0$ and $B>0$ (heat source) which enhance the heat transfer. On the other hand, the energy is absorbed by decreasing the values of $A<0$ and $B<0$ (heat sink) resulting in the temperature to drop significantly near the boundary layer. It is to be noted that the magnitude of heat transfer for temperature-dependent heat source is more pronounced compared with surface-dependent heat source. The combined effect of Soret and Dufour numbers on the temperature profiles is depicted in Fig. 10. The values of $D_{u}$ and $S_{r}$ have been selected to ensure that the product of $D_{u}$ and $S_{r}$ is constant (0.06), assuming that the mean temperature is constant. An increase in $D_{u}$ with a decrease in $S_{r}$ is used to enhance the heat transfer.

The effect of $S_{c}, K_{r}, l$ and $D_{u} \& S_{r}$ on concentration profiles are presented in Figs 11-14, respectively. Concentration profiles diminish for increasing the values of Schmidt number as shown in Fig. 11. The amplification of $S_{c}$ results in diluting molecular diffusivity of the fluid which diminishes the concentration boundary layer. To be more realistic, the values of Schmidt number are chosen to represent the diffusing chemical species of most common interest like hydrogen $\left(S_{c}=0.22\right)$, water vapor $\left(S_{c}=0.6\right)$, ammonia $\left(S_{c}=\right.$ $0.78)$ and carbon dioxide $\left(S_{c}=0.96\right)$. It is observed from Fig. 12 that the presence of a destructive chemical reaction within the boundary layer has the tendency to decrease the concentration. This is accompanied by slight decreases in the solutal boundary layer thickness and the negative wall slope of the concentration profile. Fig. 13 represents that the mass transfer increases for increasing the order of chemical reaction. Species diffusion in the boundary layer is therefore assisted with high order chemical reaction. Fig. 14 presents the combined effect of Soret and Dufour numbers on the concentration profiles. An increase in $D_{u}$ with a decrease in $S_{r}$ is used to decrease the mass transfer. 
Table 1: Effects of $\beta, K$ and $D_{u} \& S_{r}$ on $\bar{\tau}, \overline{N u}$ and $\overline{S h}$

\begin{tabular}{|c|c|c|c|c|}
\hline \multirow{2}{*}{$\begin{array}{c}\text { Physical } \\
\text { Parameter }\end{array}$} & \multirow{2}{*}{ Values } & $\overline{3}$ & \multicolumn{3}{|c|}{ Cone } \\
\cline { 3 - 5 } & & $\bar{\tau}$ & $\overline{N u}$ & $\overline{S h}$ \\
\hline \multirow{3}{*}{$\beta$} & 0.5 & 0.19780 & -0.81288 & 0.47761 \\
\cline { 2 - 5 } & 1 & 0.24137 & -0.67627 & 0.47090 \\
\cline { 2 - 5 } & 1.5 & 0.26442 & -0.62246 & 0.46836 \\
\hline \multirow{3}{*}{$K$} & 1 & 0.24137 & -0.67627 & 0.47090 \\
\cline { 2 - 5 } & 2 & 0.25658 & -0.55107 & 0.46709 \\
\cline { 2 - 5 } & 3 & 0.26307 & -0.50704 & 0.46607 \\
\hline \multirow{3}{*}{$D_{u}-S_{r}$} & $0.03-2$ & 0.18238 & 0.04383 & 0.15175 \\
\cline { 2 - 5 } & $0.05-1.2$ & 0.18314 & 0.04156 & 0.14883 \\
\cline { 2 - 5 } & $0.15-0.4$ & 0.18642 & 0.03101 & 0.15073 \\
\hline
\end{tabular}

Table 2: Effects of $A, B, K_{r}$ and $l$ on $\bar{\tau}, \overline{N u}$ and $\overline{S h}$

\begin{tabular}{|c|c|c|c|c|}
\hline \multirow{2}{*}{$\begin{array}{c}\text { Physical } \\
\text { Parameter }\end{array}$} & Values & \multicolumn{3}{|c|}{ Cone } \\
\cline { 2 - 5 } & & $\bar{\tau}$ & $\overline{N u}$ & $\overline{S h}$ \\
\hline \multirow{3}{*}{$A$} & -0.5 & 0.21708 & -0.47646 & 0.45385 \\
\cline { 2 - 5 } & 0 & 0.22500 & -0.53942 & 0.45929 \\
\cline { 2 - 5 } & 0.5 & 0.23312 & -0.60611 & 0.46499 \\
\hline \multirow{3}{*}{$B$} & -2 & 0.10064 & 1.28107 & 0.53280 \\
\cline { 2 - 5 } & 0 & 0.15311 & 0.06125 & 0.90968 \\
\cline { 2 - 5 } & 2 & 0.24710 & -2.22994 & 1.61374 \\
\hline \multirow{3}{*}{$K_{r}$} & 0 & 0.23871 & -0.60501 & 0.23181 \\
\cline { 2 - 5 } & 1 & 0.24137 & -0.67627 & 0.47090 \\
\cline { 2 - 5 }$l$ & 2 & 0.24288 & -0.72695 & 0.61615 \\
\hline \multirow{3}{*}{$l$} & 1 & 0.24110 & -0.69069 & 0.55633 \\
\cline { 2 - 5 } & 2 & 0.24137 & -0.67627 & 0.47090 \\
\cline { 2 - 5 } & 3 & 0.24120 & -0.67001 & 0.44120 \\
\hline
\end{tabular}

Figs. 15-16 indicate the variations of $\beta$ and $D_{u} \& S_{r}$ on local Skin friction against stream-wise coordinate $(X)$. Fig. 15 illustrates that local skin friction profiles increase for increasing the values of the Casson fluid parameter and magnetic field parameter. It is observed from Fig. 16 that an increase in Dufour number and a decrease in Soret number is used to diminish the magnitude of local skin-friction. Figs. 17 and 18 are plotted to show the variation of local Nusselt number profiles versus stream-wise coordinate $(X)$ for various values of $A$ and $B$. An increase in heat source parameters $(A>0 ; B>0)$ leads to decrease the rate of heat transfer whereas reverse trend is observed for decrease in heat sink parameters $(A<0 ; B<0)$. It is to be noted that $N u=\frac{\text { convective heat transfer }}{\text { conductive heat transfer }}=\frac{h L}{k}$ is physically positive number but it is negative if convective heat transfer coefficient $(h)$ is negative. This can happen since $h$ is a very poor way to describe thermo-fluid-dynamic phenomena, especially transient phenomena. In addition to that some authors have considered the negative values for length $(L)$, therefore they have obtained negative values for $N u$ number. In this manuscript, $N u$ is considered based on the references (Mohiddin et al. 2010, Muthucumaraswamy et al. 2000, Prasad et al. 2007, RushiKumar and Sivaraj, 2013, Sivaraj, R. and RushiKumar, 2013). Figs. 19 - 20 elucidate the variation of local Sherwood number against stream-wise coordinate $(X)$ for various values of $K_{r}$ and $l$. It is observed from Fig. 19 that an increase in chemical reaction parameter is used to enhance the rate of mass transfer. Fig. 20 shows that the high order chemical reactions have the tendency to diminish the local Sherwood number profiles. Table 1 shows the computed values of average skin friction coefficient, Nusselt number and Sherwood number for 
different values of $\beta, K$ and $D_{u} \& S_{r}$. The average skin friction coefficient, Nusselt number and Sherwood number for different values of $A, B, K_{r}$ and $l$ are tabulated in Table 2.

\section{Conclusion}

In this paper, we have analyzed the unsteady Casson fluid flow over a vertical cone with the influence of crossdiffusion, non-uniform heat source/sink and high order chemical reaction. Some significant findings of this investigation are summarized as follows:

- An increase in Casson fluid parameter decreases the fluid flow whereas the reverse trend is observed for porous permeability parameter.

- Higher values of Prandtl number have the tendency to reduce the temperature profiles.

- The heat transfer characteristics of the fluid strongly depend on the temperature dependent heat source/sink as well as surface dependent heat source/sink.

- An increase in Dufour number with a decrease in Soret number is to increase the heat transfer on the cone while the trend is reversed in the case of mass transfer.

- The concentration profiles fall for increasing the Schmidt number and chemical reaction parameter.

- An increase in order of chemical reaction parameter results in diluting the influence of chemical reaction parameter thereby increasing mass transfer profiles. This shows that heavier diffusing species have greater retarding effect in concentration distribution.

\section{References}

Abolbashari, M. H., Freidoonimehr, N., Nazari, F. and Rashidi, M. M. (2015): Analytical modeling of entropy generation for casson nano-fluid flow induced by a stretching surface, Advanced Powder Technology, Vol. 26, pp. 542-552. http://dx.doi.org/10.1016/j.apt.2015.01.003

Alam, M. S., Rahman, M. M. and Sattar, M. A. (2009): Transient magnetohydrodynamic free convective heat and mass transfer flow with thermophoresis past a radiate inclined permeable plate in the presence of variable chemical reaction and temperature dependent viscosity, Nonlinear Analysis Modeling and Control, Vol. 14, pp. 3-20.

Animasaun, I. L. (2015): Effects of thermophoresis, variable viscosity and thermal conductivity on free convective heat and mass transfer of non-darcian MHD dissipative Casson fluid flow with suction and nth order of chemical reaction, Journal of the Nigerian Mathematical Society, Vol. 34, No. 1, pp. 11-31. http://dx.doi.org/10.1016/j.jnnms.2014.10.008

Mohiddin, S. G., Prasad, V. R., Varma, S. V. K., and Anwar Beg, O. A. (2010): Numerical study of unsteady free convective heat and mass transfer in a Walters-B viscoelastic flow along a vertical cone, International Journal of Applied Mathematics and Mechanics Vol. 6, No. 15, pp. 88-114.

Mukhopadhyay, S. and Vajravelu, K. (2013): Diffusion of chemically reactive species in Casson fluid flow over an unsteady permeable stretching surface, Journal of Hydrodynamics, Vol. 25, No. 4, pp. 591-598. http://dx.doi.org/10.1016/S1001-6058(11)60400-X

Muthucumaraswamy, R. and Ganesan, P. (2000): Flow past an impulsively started vertical plate with constantheat flux and mass transfer, Computer Methods in Applied Mechanics and Engineering, Vol. 187, pp. 79-90.

Nandeppanavar, M. M., Vajravelu, K. and Abel, M. S. (2011): Heat transfer in MHD viscoelastic boundary layer flow over a stretching sheet with thermal radiation and non-uniform heat source/sink, Communications in Nonlinear Science and Numerical Simulation, Vol. 16, No. 9, pp. 3578-3590. http://dx.doi.org/10.1016/j.cnsns.2010.12.033

Okedoye, A. M. (2014): Unsteady MHD mixed convection flow past an oscillating plate with heat source/sink, Journal of Naval Architecture and Marine Engineering, Vol. 11, pp. 167-176. http://dx.doi.org/10.3329/jname.v11i2.6477

Pal, D. and Mondal, H. (2010): Effect of variable viscosity on MHD non-Darcy mixed convective heat transfer over a stretching sheet embedded in a porous medium with Non-uniform heat source/sink, Communications in Nonlinear Science and Numerical Simulation, Vol. 15, No. 6, pp. 1553-1564. http://dx.doi.org/10.1016/j.cnsns.2009.07.002 
Postelnicu, A. (2004): Influence of a magnetic field on heat and mass transfer by natural convection from vertical surfaces in porous media considering Soret and Dufour effects, International Journal of Heat and Mass Transfer, Vol. 47, No. 6-7, pp. 1467-1472. http://dx.doi.org/10.1016/j.ijheatmasstransfer.2003.09.017

Prasad, V. R., BhaskarReddy, N. and Muthucumaraswamy, R. (2007): Radiation and mass transfer effects on two-dimensional flow past an impulsively started infinite vertical plate, International Journal of Thermal Sciences, Vol. 46, pp. 1251-1258.

Prasad, V. R., Rao, A. S., Reddy, N. B. and Beg, O.A. (2013): Modelling laminar transport phenomena in a Casson rheological fluid form an isothermal sphere with partial slip in a non- Darcy porous medium, Journal of Theoretical and Applied Mechanics, Vol. 40, No. 4, pp. 469-510. http://dx.doi.org/10.2298/TAM1304469P

Raju, M. C. and Varma, S. V. K. (2014): Soret effects due to natural convection in a non-Newtonian fluid flow in porous medium with heat and mass transfer, Journal of Naval Architecture and Marine Engineering, Vol. 11, pp. 147-156. http://dx.doi.org/10.3329/jname.v11i2.17563

RamReddy, C., Murthy, P. V. S. N., Chamkha, A. J. and Rashad, A. M. (2013): Soret effect on mixed convection flow in a nano-fluid under convective boundary condition, International Journal of Heat and Mass Transfer, Vol. 64, pp. 384-392. http://dx.doi.org/10.1016/j.ijheatmasstransfer. 2013.04.032

Rashidi, M. M., Rahimzadeh, N., Ferdows, M., Uddin, M. J. and Beg, O. A. (2012): Group theory and differential transform analysis of mixed convective heat and mass transfer from a horizontal surface with chemical reaction effects, Chemical Engineering Communication, Vol. 199, No. 8, pp. 1012-1043. http://dx.doi.org/10.1080/00986445.2011.636850

Rahman, M. M., Uddin, M. J. and Aziz, A. (2009): Effects of variable electric conductivity and non-uniform heat source (or sink) on convective micropolar fluid flow along an inclined flat plate with surface heat flux, International Journal of Thermal Sciences, Vol. 48, pp. 2331-2340. http://dx.doi.org/10.1016/j.ijthermalsci.2009.05.003

Rahman, M. M. and Al-Lawatia, M. (2010): Effects of higher order chemical reaction on micropolar fluid flow on a power law permeable stretched sheet with variable concentration in a porous medium, The Canadian Journal of Chemical Engineering, Vol. 88, No. 1, pp. 23-32. http://dx.doi.org/10.1002/cjce.20244

RushiKumar, B. and Sivaraj, R. (2013): Heat and mass transfer in MHD viscoelastic fluid flow over a vertical cone and flat plate with variable viscosity, International Journal of Heat and Mass Transfer, Vol. 56, pp. 370379. http://dx.doi.org/10.1016/j.ijheatmasstransfer. 2012.09.001

RushiKumar, B. and Sivaraj, R. (2013): MHD viscoelastic fluid non-Darcy flow over a vertical cone and a flat plate, International Communications in Heat and Mass Transfer, Vol. 40, pp. 1-6, http://dx.doi.org/10.1016/j.icheatmasstransfer.2012.10.025

Sivaraj, R. and RushiKumar, B. (2013): Chemically reacting dusty viscoelastic fluid flow in an irregular channel with convective boundary, Ain Shams Engineering Journal, Vol. 4, pp. 93-101. http://dx.doi.org/10.1016/j.asej.2012.06.005

Sivaraj, R. and RushiKumar, B. (2013): Viscoelastic fluid flow over a moving vertical cone and flat plate with variable electric conductivity, International Journal of Heat and Mass Transfer, Vol. 61, pp. 119-128. http://dx.doi.org/10.1016/j.ijheatmasstransfer.2013.01.060 